\title{
Real-world data on the effectiveness and safety of interferon- alpha-2a intralesional injection for the treatment of focally recalcitrant mycosis fungoides
}

\author{
Shi-Yu Zhang, Zhao-Rui Liu, Lu Yang, Tao Wang, Jie Liu, Yue-Hua Liu, Kai Fang \\ Department of Dermatology, Peking Union Medical College Hospital, Peking Union Medical College and Chinese Academy of Medical Sciences, \\ Beijing, China \\ Contributions: (I) Conception and design: T Wang, YH Liu; (II) Administrative support: J Liu, YH Liu; (III) Provision of study materials or patients: \\ T Wang; (IV) Collection and assembly of data: SY Zhang, ZR Liu, L Yang; (V) Data analysis and interpretation: SY Zhang, K Fang; (VI) Manuscript \\ writing: All authors; (VII) Final approval of manuscript: All authors. \\ Correspondence to: Yue-Hua Liu. Department of Dermatology, Peking Union Medical College Hospital, Peking Union Medical College and Chinese \\ Academy of Medical Sciences, No. 1 Shuaifuyuan, Wangfujing, Beijing, China. Email: yuehualiu63@163.com.
}

Background: Focally recalcitrant mycosis fungoides (MF) is challenging to treat. Despite interferon
(IFN)- $\alpha$ intralesional injection having been tested in randomized controlled trials for the treatment of patch
or early plaque lesions of MF, no real-world data regarding this therapy for focally recalcitrant MF has been
reported. This study aimed to evaluate the effectiveness and safety of IFN- $\alpha-2$ a intralesional injection in
focally recalcitrant MF.

Methods: Data on all cases of focally recalcitrant MF treated by IFN- $\alpha$-2a intralesional injection in Peking Union Medical College Hospital from January 1, 2015 to December 31, 2019 were retrospectively retrieved. Based on clearance of injected lesions and the proportion of adverse events (AEs), the effectiveness and safety of the treatment were analyzed, respectively.

Results: Of the 15 patients included, 10 (66.7\%) achieved complete response (CR), and 1 (6.7\%) patient had partial response. The overall response rate was $73.3 \%$. The clearance rate of lesions in the sun-exposed areas was significantly higher than that in the non-sun-exposed areas $(\mathrm{P}<0.01)$. The $\mathrm{CR}$ rate in patients with disease duration of less than 20 years was significantly higher than that in the patients with disease duration of at least 20 years $(\mathrm{P}<0.05)$. Four $(26.7 \%)$ patients had stable disease (SD). The median event-free survival (EFS) was 4.0 months (95\% CI, 1.8-6.1 months; range, 1-28+ months). There were no severe acute or chronic side effects.

Conclusions: IFN- $\alpha-2$ a intralesional injection is an effective and safe treatment modality for the treatment of focally recalcitrant MF.

Keywords: Interferon; intralesional injection; mycosis fungoides (MF); therapy

Submitted Feb 09, 2020. Accepted for publication Jul 09, 2020.

doi: $10.21037 /$ atm-20-1458

View this article at: http://dx.doi.org/10.21037/atm-20-1458

\section{Introduction}

Mycosis fungoides (MF) is an indolent T cell nonHodgkin's lymphoma that primarily affects the skin but has potential for systemic neoplastic infiltration of the lymph nodes, blood, and viscera. The pathogenesis of MF is unclear. Genetic and epigenetic abnormalities have been identified in MF tumor cells (1-3), and cytomegalovirus and Epstein-Barr virus have both been associated with the development of MF $(4,5)$. Studies have also suggested that in MF patients, increased T-helper type 2 (TH2) activity and decreased numbers and activity of both NK cells and CD8+ T cells weaken the host's immune activity, thus 
contributing to the development of MF (6-8). Although early-stage MF lesions can generally be well-controlled with current therapies, advanced lesions usually show a much poorer response. Moreover, a curative effect is still not possible for some patients with relapsed/refractory diseases.

Interferons are naturally occurring polypeptides produced via eukaryotic cell stimulation. As a part of the innate immune response, interferons have been shown to have antivirus, antiproliferative, and immunomodulating effects. As well as restraining neoplasm proliferation through both the inhibition of multiple growth factors and the regulation of oncogene and anti-oncogene expression, interferons also reduce IL-4 and IL-5 production to suppress the problematic increased TH2 activity (9), which makes interferons particularly helpful for treating MF. Recent studies have revealed that IFN- $\alpha-2$ a modulates the production of chemokines including CCL17, CCL18, CXCL10, and CXCL11 from tumor-associated macrophages (10), a group of macrophages which recruit TH2 and tumor cells at the lesional skin of cutaneous T cell lymphoma (CTCL) $(11,12)$. IFN- $\alpha$ administered as an intramuscular or subcutaneous injection has been proven in numerous clinical studies to be effective either alone or when used in combination with other therapies, including psoralen plus ultraviolet A (PUVA), bexarotene, and total skin electron beam therapy (TSEBT) (13-16). Interferon intralesional injection has been reported to achieve a beneficial effect in MF and other CTCL patients in smallerscale studies (17-19). However, data on the application of interferon intralesional injection in refractory MF patients are limited. The goal of this retrospective study was therefore to assess the effectiveness and safety of IFN- $\alpha-2 \mathrm{a}$ intralesional injection in focally recalcitrant MF patients. We present the following article in accordance with the STROBE reporting checklist (available at http://dx.doi. org/10.21037/atm-20-1458).

\section{Methods}

\section{Patients and data collection}

After the study was approved by the Institutional Review Board of Peking Union Medical College Hospital, Peking Union Medical College and Chinese Academy of Medical Sciences, electronic health records were retrospectively queried to generate a list of patients who had $\geq 1 \mathrm{MF}$ lesions refractory to previous treatment for $\geq 6$ months and who were administered at least 1 dose of IFN- $\alpha-2$ a intralesional injection at Peking Union Medical College Hospital between January 1, 2015 and December 31, 2019. Patient information including gender, age at onset, age at baseline, clinical photography of all injected lesions, lesion number and size, records of symptoms, disease stage, previous treatment for the refractory lesions, and the results of complete blood count and liver function tests before therapy, every other week during therapy, and 1 and 2 weeks after therapy, was collected and analyzed. All procedures of interferon intralesional injection were performed without general anesthesia at the outpatient clinic. The study was conducted in accordance with the Declaration of Helsinki (as revised in 2013). The study was approved by institutional ethics board of Peking Union Medical College Hospital (No. S-k357) and informed consent was taken from all the patients. The study was performed in accordance with approved guidelines and regulations.

\section{Measurements}

Lesion size was defined as the area of the lesion, and was computed as maximum length $(\mathrm{cm}) \times$ maximum width $(\mathrm{cm})$. The skin response was defined as the response of the intralesionally injected lesions during the therapy, and the response was rated according to Olsen et al., as follows: complete response (CR), 100\% clearance of injected lesions; partial response (PR), 50-99\% clearance of skin disease from baseline without new tumors $\left(\mathrm{T}_{3}\right)$ in patients with $\mathrm{T}_{1}, \mathrm{~T}_{2}$, or $\mathrm{T}_{4}$ only skin disease; stable disease (SD), $<25 \%$ increase to $<50 \%$ clearance in skin disease from baseline without new tumors $\left(T_{3}\right)$ in patients with $T_{1}, T_{2}$, or $T_{4}$ only skin disease; progressive disease (PD), $>25 \%$ increase in skin disease from baseline, or new tumors $\left(\mathrm{T}_{3}\right)$ in patients with $\mathrm{T}_{1}, \mathrm{~T}_{2}$ or $\mathrm{T}_{4}$ only skin disease, or loss of response (increase in skin score greater than the sum of nadir plus $50 \%$ baseline score in those with complete or partial response); or relapse, any disease recurrence in those with CR (20).

\section{Definitions}

An event was considered to be disease progression, relapse, discontinuation of IFN- $\alpha-2$ a intralesional injection without $100 \%$ clearance of the injected lesions, or death.

Event-free survival (EFS) was considered to be the time from the initiation of IFN- $\alpha-2$ a intralesional injection treatment to disease progression, relapse, discontinuation of IFN- $\alpha$-2a intralesional injection without $100 \%$ clearance of the injected lesions, death from any cause, or last follow-up. 
Table 1 Patient characteristics $(n=15)$

\begin{tabular}{lc}
\hline Characteristics & No. of patients \\
\hline Age (years) & $46.9[28-62]$ \\
Mean [range] & \\
Sex, n (\%) & $4(26.7)$ \\
Male & $11(73.3)$ \\
Female & \\
Stage, n (\%) & $12(80.0)$ \\
IIA & $3(20.0)$ \\
IIB & \\
Disease duration (years) & $17.9[7-49]$ \\
Mean [range] & \\
Total dose (million IU) & $75.8[18-108]$ \\
Mean [range]
\end{tabular}

IU, international unit.

\section{Data analysis}

Statistical analyses were performed using SPSS 23.0 (IBM, NY, U.S.) software. Where appropriate, $\chi^{2}$ or Fisher's exact test were used to compare proportions. In all cases, a $\mathrm{P}$ value $<0.05$ was considered statistically significant. EFS were estimated by the Kaplan-Meier method. Missing data were handled using regression with SPSS 23.0 software.

\section{Results}

\section{Patient characteristics}

A total of 15 patients (4 males and 11 females) with focally recalcitrant MF lesions treated with IFN- $\alpha-2$ a intralesional injection were identified. Patient characteristics are shown in Table 1. The patients had a mean age of $46.9 \pm$ 11.6 years (range, 28-62 years) and a mean disease duration of 17.9 years (range, 7-49 years). All patients were staged according to Olsen et al. (20). The stages of disease ranged from IIA (12 patients) to IIB (3 patients). All of the patients were treated with the following procedure: before the injection, $3 \times 10^{6}$ units of recombinant IFN- $\alpha-2$ a $\left(\right.$ Intefen ${ }^{\circledR}$; 3 SBio Inc., Shenyang, China) were dissolved in $2 \mathrm{~mL}$ saline. The intralesional injection was performed using a 23 -gauge needle 3 times a week. For patients with more than 1 lesion needing treatment, each lesion was given a dose proportional to its size. The injection sites were locally compressed for 5 minutes after the injection. The mean treatment time was $58.7 \pm 25.9$ days, and the median treatment time was 70.0 days. The mean total number of treatment sessions was $25.3 \pm 11.1$. The mean total dose was $75.8 \pm 33.3 \mathrm{MU}$. Throughout the interferon intralesional injection treatment period, all patients kept undergoing ultraviolet A phototherapy and/or narrow band ultraviolet B (NB-UVB) phototherapy which they had been treated with before this study. A total of $41 \mathrm{MF}$ lesions received interferon intralesional injection. There were no distant lesions which were not directly injected throughout the period of this therapy. The most common sites of the lesions were the thigh (11 lesions), abdomen (6 lesions), and foot (6 lesions). The total lesion size was $\leq 5$ and $\leq 30 \mathrm{~cm}^{2}$ in $6(40.0 \%)$ and 13 patients $(86.7 \%)$, respectively. Prior treatment modalities to which the lesions most commonly showed resistance included phototherapy, oral acitretin, and intramuscular interferon; 9 patients $(60.0 \%)$ failed $\geq 3$ therapies, 6 of whom failed phototherapy, intramuscular interferon, and oral acitretin. The mean follow-up time from the first interferon intralesional injection was 18.9 months (range, 4-32 months; median, 20 months). Parts of the data for lesions size during therapy were missing in 2 patients.

\section{Effectiveness}

As illustrated in Table 2, a marked decrease in the size of the interferon-injected MF lesions was observed. After the 12th week, 10 (66.7\%) patients had achieved CR. A total of 28 lesions (including 1 lesion of patient 12 with SD) cleared completely, with 3 lesions improving markedly and 7 lesions having less than $50 \%$ reduction in lesion size. Representative photographs of the lesions before and after injection are shown in Figure 1 (patient 4) and Figure 2 (patient 7). Patient 4 reached CR after 4 weeks of treatment (Figure 1), while patient 7 achieved CR after 10 weeks of treatment (Figure 2). Each of the 13 lesions (13/13, 100\%) in the sun-exposed areas including the head, arm, and hand, had a complete clearance. Of the 28 lesions in the non-sun-exposed areas, $15(15 / 28,53.6 \%)$ had a complete clearance, which was significantly fewer than in the sunexposed areas $(\mathrm{P}<0.01)$. One $(6.7 \%)$ patient reached $\mathrm{PR}$. The overall response rate was $73.3 \%$. Furthermore, 8 in 10 patients $(8 / 10,80 \%)$, aged 50 years old or younger at the initiation of intralesional injection achieved CR, while 2 in 5 patients (40\%) older than 50 years old achieved CR. The difference in CR rate between the two age groups 


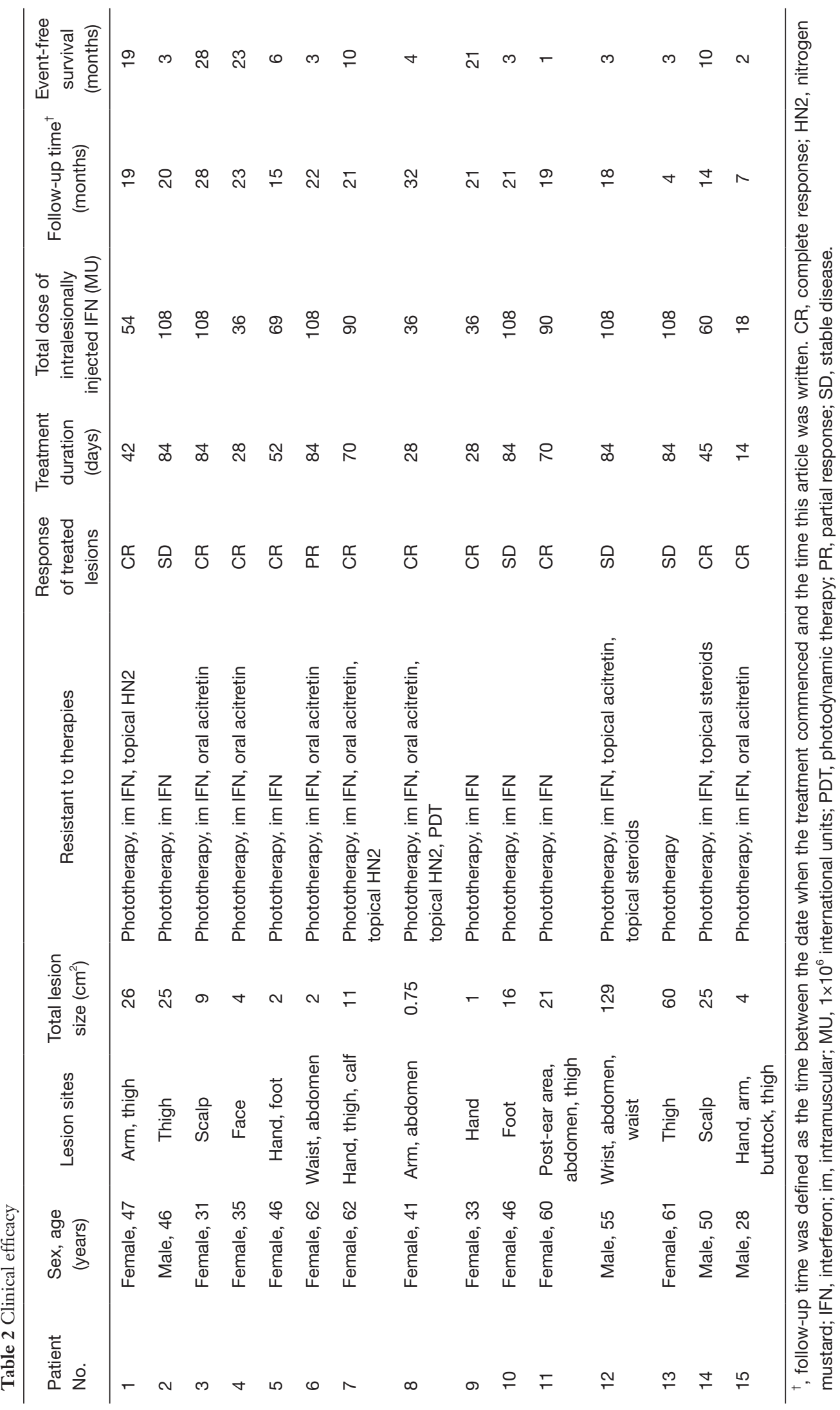



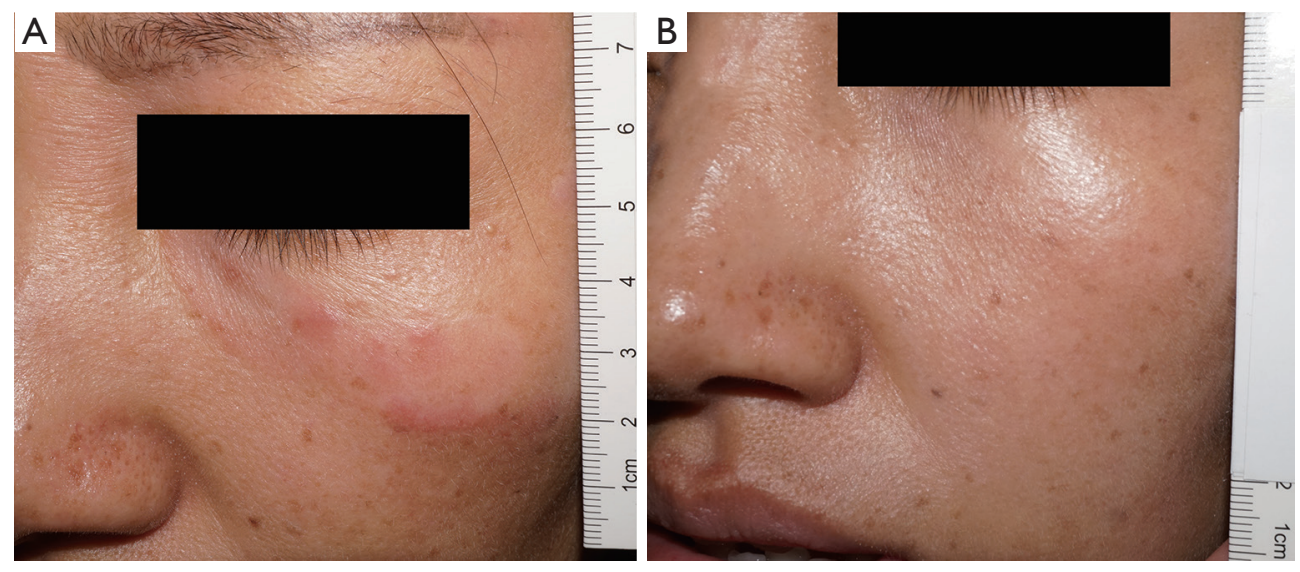

Figure 1 Left cheek of patient 4 before (A) and after (B) 4 weeks of interferon intralesional injection. The patient is a 35 -year-old woman diagnosed with stage IIA mycosis fungoides who was treated with PUVA, intramuscular interferon, and oral acitretin. The lesion on the left cheek appeared and persisted despite of the treatment. After application of intralesional interferon for 4 weeks, the lesion cleared completely (45 $\mathrm{mm} \times 25 \mathrm{~mm}$ before intralesional injection, $0 \mathrm{~mm} \times 0 \mathrm{~mm}$ after 4 weeks of intralesional injection treatment).
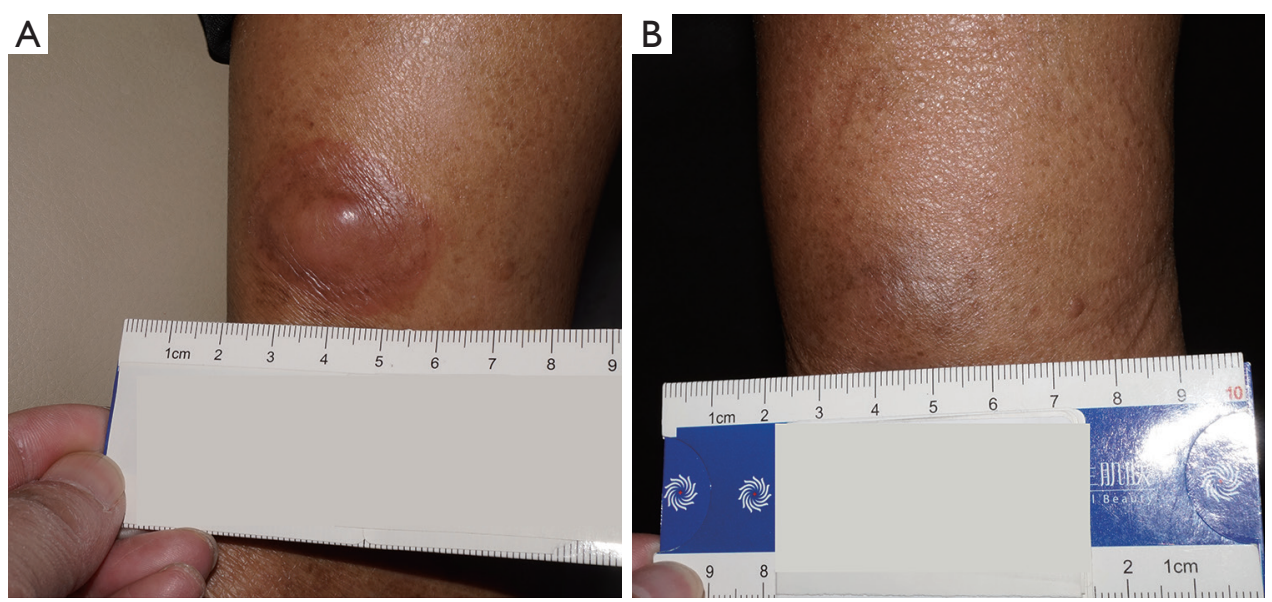

Figure 2 Right forearm of patient 7 before (A) and after (B) 10 weeks of interferon intralesional injection. The patient is a 62 -year-old woman diagnosed with stage IIB mycosis fungoides and was treated with PUVA, nitrogen mustard, intramuscular interferon, and oral acitretin. The lesion on the right forearm persisted despite the treatment. After application of intralesional interferon for 10 weeks, the lesion had completely cleared ( $35 \mathrm{~mm} \times 35 \mathrm{~mm}$ before intralesional injection, $0 \mathrm{~mm} \times 0 \mathrm{~mm}$ after 10 weeks of intralesional injection).

was not statistically significant $(\mathrm{P}=0.25)$. The $\mathrm{CR}$ rate of patients whose disease duration was less than 20 years at the initiation of intralesional injection was $83.3 \%(10 / 12)$, which was significantly higher than that of patients with a disease duration of at least 20 years $(0 / 3,0 \%, \mathrm{P}<0.05)$. CR was reached within 8 weeks in 5 of the 6 patients with a total lesion size of $\leq 5 \mathrm{~cm}^{2}$. However, there was no statistically significant difference in CR rate found between patients with a total lesion size of $\leq 5$ or $>5 \mathrm{~cm}^{2}(\mathrm{P}=0.58)$. CR was reached within 12 weeks in 10 of the 13 patients with a total lesion size of $\leq 30 \mathrm{~cm}^{2}$, while both patients with total lesion size of $>30 \mathrm{~cm}^{2}$ had SD. Of the 12 IIA stage patients, 7, 1, and 4 patients had CR, PR, and SD, respectively. All three IIB stage patients had CR. By the end of the 12th week, 7 of the 9 patients who had failed $\geq 3$ therapies had achieved CR. Among the 6 patients who had no response to treatment of phototherapy, intramuscular interferon, and oral acitretin, 5 achieved CR, and 1 had PR. Four (26.7\%) patients had SD. There was no PD during the period of interferon intralesional injection. One injected lesion on the 


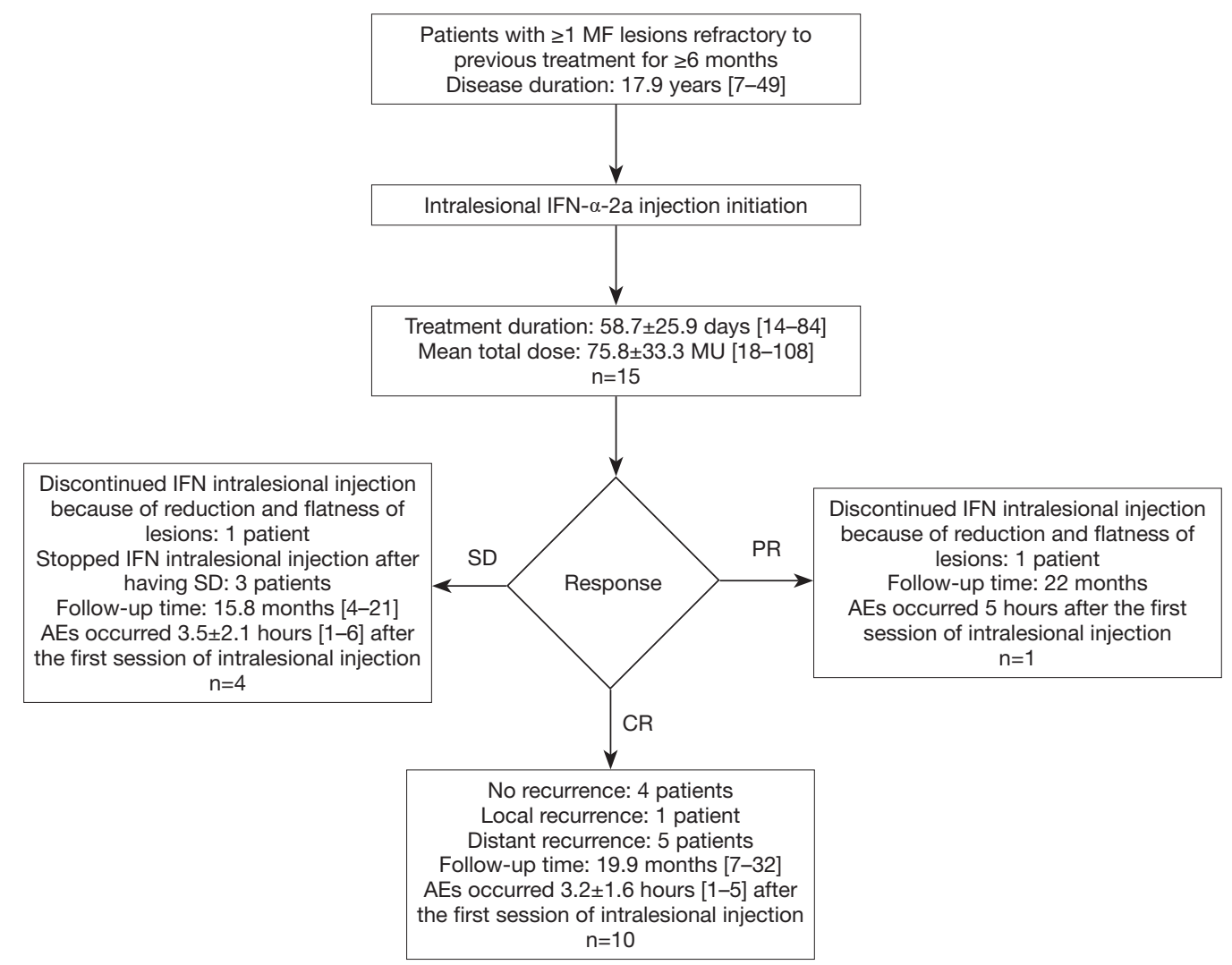

Figure 3 Event flow diagram of the disease history, interferon intralesional injection initiation and duration, and AE occurrence for the 15 patients. AE, adverse event; CR, complete response; IFN, interferon; MF, mycosis fungoides; MU, $1 \times 10^{6}$ international units; PR, partial response; $\mathrm{SD}$, stable disease.

left calf of patient 7 had local recurrence 10 months after its first clearance, and regressed completely after the same regimen of interferon intralesional injection for 2 weeks. There has been no local recurrence in the other patients as of the writing of this article. In patients who had $100 \%$ clearance of injected lesions, 5 patients (patients 5, 8, 11, 14 , and 15) developed new lesions in other sites not treated with intralesional interferon $6,4,1,10$, and 2 months after treatment began, respectively. In the 11 patients with symptoms including pruritus and pain, the symptoms of 7 (63.7\%) patients were completely relieved, and 3 (27.2\%) patients showed significant improvement.

Table 2 shows the EFS of each patient. Among the 11 patients whose events were defined in the Methods section, patient 6 and 10 discontinued interferon intralesional injection after their lesions were significantly reduced and flattened (despite not meeting the criteria of CR), and had continued maintenance treatment of NB-UVB, which led to gradual clearance of the injected lesions with no relapse or progression having occurred at the time of this writing; patients 2,12, and 13 stopped interferon intralesional injection and had other treatment (patient 2, electron beam therapy; patients 12 and 13, intramuscular interferon) after having SD; patient 7 had local recurrence; patients 5, 8, 11, 14, and 15 developed new lesions in distant sites. The median EFS was 4.0 months (95\% CI, 1.8-6.1 months; range, 1-28+ months). All of the patients who relapsed $(60 \%, 6 / 10)$ were patients with CR. The median EFS of these relapsed patients was 5 months (range, 1-10 months). Results for the 15 patients are shown in Figure 3.

\section{Safety and tolerability}

A total of 49 drug-related AEs were reported in all of the patients enrolled. The most common drug-related AEs were influenza-like symptoms including fever (86.7\%) and fatigue $(66.7 \%)$. These symptoms occurred $3.4 \pm$ 1.7 hours (range, 1-6 hours; median, 4 hours) after the 
first session of interferon intralesional injection, and were improved with oral acetaminophen. These symptoms were markedly relieved and gradually disappeared during the course of the therapy. Mild and transient fever occurred in 13 patients. Four patients reported that the severity of symptoms associated with interferon intralesional injection were milder than those of intramuscular injection at the same dose. Furthermore, 8 (53.3\%) patients experienced mild hair loss, 2 patients had slightly elevated levels of liver function enzyme, and 1 patient experienced mild and transient leukocytopenia. These lab test abnormalities occurred during the treatment and returned to normal levels in 2 weeks after the last intralesional injection. There were no cases of severe AEs.

\section{Discussion}

From January 1, 2015 to December 31, 2019, 15 patients (4 males and 11 females) with focally recalcitrant MF were treated with interferon intralesional injection in Peking Union Medical College Hospital. IFN- $\alpha-2$ a was administered topically by intralesional injection into lesions at a dose of $3 \mathrm{MU}, 3$ times per week for up to 12 weeks.

Based on the response evaluation criteria for $\mathrm{MF}(20)$, the overall response rate was $73.3 \%$ (11/15, CR rate of $66.7 \%)$; $100 \%(6 / 6,5 \mathrm{CR}, 1 \mathrm{PR})$ for patients with total lesion size of $\leq 5 \mathrm{~cm}^{2}, 71.4 \%(5 / 7,5 \mathrm{CR})$ for patients with total lesion size between 5 and $30 \mathrm{~cm}^{2}$, and $0 \%(0 / 2)$ for patients with total lesion size of $\geq 30 \mathrm{~cm}^{2}$. These CR rates suggest the CR rates might be negatively associated with total lesion size, although no statistically significant differences were found. This point needs to be further investigated in largerscale studies. In a total of 41 intralesionally injected lesions, $28(68.3 \%)$ cleared completely, $3(7.3 \%)$ showed marked improvement, and $7(17.1 \%)$ had less than $50 \%$ reduction in lesion size. Lesions in sun-exposed areas had a significantly higher rate of clearance than those in non-sun-exposed areas $(100 \%$ vs. $53.6 \%, \mathrm{P}<0.01)$, which might be attributed to the larger amount of ultraviolet exposure in sun-exposed skin. The CR rate was significantly higher in patients with a disease duration of less than 20 years than in those with MF for at least 20 years when intralesional injection began (83.3\% vs. $0 \%, \mathrm{P}<0.05)$, which suggests that the therapy might be more effective when used earlier. The advanced immune suppression in late-stage MF might be responsible for the loss of the IFN effect. Intefen ${ }^{\circledR}$ was a previously marketed IFN- $\alpha-2$ a product for the treatment of lymphoma. The outcome of this study was consistent with a previous study on IFN- $\alpha$ intralesional injection to treat MF (19). According to Wolff et al., all 9 lesions injected with IFN- $\alpha$ showed significant response, and the average degree of improvement was above moderate improvement. Together, these findings revealed that interferon intralesional injection is an effective therapy for MF.

Intralesional injection has long been an important dermatologic therapy, which can be effectively and safely used in the treatment of many diseases. Intralesional injection delivers the medication directly into the target lesions, meaning the concentration of medication in the lesion remains localized and elevated. Moreover, intralesional injection makes the skin a reservoir which releases the deposited medication over a period of time. In these ways, intralesional injection leads to better, prolonged response, while minimizing systemic side effects. According to Liu et al. (21), intralesional corticosteroids were safe and effective for the treatment of 4 patients with focally resistant MF. The successful use of intralesional injection in both Liu's study and in the current study supports it as an ideal method of administration in treatment of focally resistant MF.

Interferon has been widely used alone or in combination with other therapies to treat MF. Various functions, including anti-virus, anti-proliferation, cytotoxicity, and immunomodulation, contribute to the efficacy of interferon in the treatment of MF $(9,22)$. Intramuscular and subcutaneous injections are the most common route of administration. Jumbou et al. reported that 41\% (21/51) patients with MF and Sezary syndrome (SS) treated with subcutaneous IFN- $\alpha-2$ a achieved CR (13). Papa et al. reported that subcutaneous IFN- $\alpha-2$ a led to an OR rate of $66.7 \%(10 / 15)$ in relapsed or refractory MF and SS patients (23). According to Hüsken et al., subcutaneous IFN- $\alpha-2$ a plus oral PUVA led to CR in $37.5 \%$ (3/8) patients and PR in $12.5 \%(1 / 8)$ patients (24), which was consistent with our results that showed the synergistic therapeutic effect of interferon intralesional injection and ultraviolet phototherapy. Low-dose systemic interferon therapy has also been demonstrated to be effective and safe in the treatment of CTCL $(9,14,15,22)$. In advanced-stage MF, low-dose systemic IFN- $\alpha$-2a maintenance treatment was reported to be beneficial in controlling relapse following autologous stem cell transplantation (25). The advantages of low-dose systemic interferon include better tolerance and lower cost, which make the therapy an effective, welltolerated, and well-adhered-to option for maintaining durable remission $(15,25)$. 
Intralesional interferon injection maintains a localized, elevated, and prolonged concentration of interferon in the lesion, which leads to some of the advantages of this therapy. The advantages of intralesional interferon in treatment for focally recalcitrant MF lesions include a greater therapeutic effect, and the reduction of the dose of interferon compared to systemic interferon therapy. The effectiveness of intralesionally injected interferon in MF patients has been described in earlier randomized controlled trials and small-scale case studies. Wolff $e t a l$. reported that $100 \%$ (9/9) of patch- or early plaque-phase MF lesions improved after IFN- $\alpha$ intralesional injection of $2 \mathrm{MU}, 3$ times per week for 4 weeks (19). According to Vonderheid et al., $83.3 \%$ (10/12) of plaque-phase MF lesions treated with IFN- $\alpha-2 b$ intralesional injection of $1 \mathrm{MU}$ per site, 3 times per week for 4 weeks reached complete clinical regression, and $16.7 \%(2 / 12)$ had marked improvement (18). In the lesions injected in our study, $92.7 \%(38 / 41)$ showed complete or partial improvement, which is lower than the rates achieved by Wolff et al. $(100 \%, 9 / 9)$ and Vonderheid et al. (100\%, 12/12). However, the dose used in our study was higher than the dose in the studies by Wolff et al. and Vonderheid et al. These differences in efficacy and dose might have resulted from the differences in the stage of MF (12 IIA, 3 IIB vs. 6 IA, 3 IB and 1 IA, $1 \mathrm{IB}, 4 \mathrm{IIA})$ and disease duration (17.9 years vs. 9.55 and 36.3 months). In addition, the lesions in our study were refractory to previous therapies for $\geq 6$ months, and might have had stronger resistance to treatment, thus leading to a difference in efficacy. Qiu et al. reported application of intralesional IFN- $\alpha-2 b 1 \mathrm{MU}$, once a week in $3 \mathrm{MF}$ patients (2 IIA stage, 1 IIB stage) whose lesions were resistant to intramuscular interferon. Intralesional interferon was used for 3 to 6 weeks, together with the continuation of the intramuscular interferon regimen, and led to complete clearance of the lesions. After the dosage was gradually decreased and discontinued, the progression-free survival was 2 months, 1 month, and 2 months, respectively (26). In our study, $68.3 \%(28 / 41)$ of the injected lesions had complete resolution, which is a lower rate than that of the study by Qiu et al. (100\%, 3/3). This difference suggests that intralesional interferon used in combination with intramuscular interferon might be more efficacious and warrants further verification by large-scale studies. According to $\mathrm{Hu}$ et al., $2 \mathrm{MF}$ patients who had refractory lesions treated with recombinant human leukocyte IFN- $\alpha 2$ showed a marked response in 8 and 3 months, respectively, and eventually achieved complete resolution of the treated lesions. The dose was $0.5 \mathrm{MU} 3$ times per week at the beginning and was later increased to $1.0 \mathrm{MU} 3$ times per week (27). The time of intralesional interferon treatment to reach significant response was longer in our and $\mathrm{Hu}$ et al.'s study than in the studies by Wolff et al. and Vonderheid et al., which suggests that refractory MF lesions might need prolonged intralesional interferon treatment with a higher total dose. Yamamoto et al. reported a case study in which intralesional recombinant IFN- $\gamma$ in 2 patients with MF led to improvement, and patients were free of recurrence for 4 and 3 weeks (28). Because only a small number of studies have been conducted on intralesional IFN- $\gamma$ in MF, large-scale clinical trials and case studies are needed to investigate its effectiveness and safety and to draw comparisons with intralesional IFN- $\alpha$. In a prospective study, adenoviral vector containing IFN- $\gamma$ complementary DNA was intralesionally injected each week for up to 12 cycles (each cycle comprised 3 weeks with injections and 1 week without an injection) with a total dose of $3 \times 10^{11}$ total particles in $10 \mathrm{MF}$ patients. The injected lesions had $\mathrm{PR}, \mathrm{SD}$, and PD in 4, 3, and 3 patients, respectively (29). This therapy, which functions by local secretion instead of immediate injection of interferon, provides new potential options for intralesional interferon. As was revealed in the previous study and in our present study, the drug delivery route of intralesional injection significantly enhanced the antitumor activities of interferon within both early-phase and refractory MF lesions, leading to marked response.

The effect of intralesional administration in reducing the dose of interferon has also been described in previous literature. The doses of interferon reported by Wolff et al. and Vonderheid et al. were lower than the lowest clinically relevant systemic dose (3 MU, 3 times per week). The outcomes of these studies indicate that intralesional injection could reduce the dose of interferon. The doses of intralesional interferon in all patients in our study were equal to the lowest clinically relevant systemic dose, which were higher than the doses described by $\mathrm{Hu}$ et al. (3 MU, 3 times per week, vs. 0.5-1.0 MU, 3 times per week); however, treatment time tended to be shorter in our study than in $\mathrm{Hu}$ et al.'s report. These differences suggest that intralesional interferon with doses lower than the lowest clinically relevant systemic doses is efficacious in focally recalcitrant MF, but longer treatment times may be needed in turn, and should thus be verified in larger clinical studies. The advantage of intralesional interferon injection in treating recalcitrant MF lesions with low doses (3 MU, 3 times per week or lower) might make the therapy 
an alternative application of low dose systemic interferon in focally refractory MF. Further investigations, especially randomized controlled clinical trials, are needed to establish the lowest effective dose of intralesional interferon in focally recalcitrant $M F$.

Wolff et al. (19) and Vonderheid et al. (18) reported that intralesionally injected interferon could lead to detectable improvement of distant lesions which were not directly injected. This might have resulted from systemic absorption of interferon. However, the patients in our study had all the refractory lesions intralesionally injected and there was no distant lesion. Therefore, the response of distant lesions could not be assessed in the present study. Larger-scale randomized controlled clinical studies are needed to further investigate the distant therapeutic effect of intralesional interferon in focally recalcitrant $\mathrm{MF}$.

$\mathrm{MF}$ is an indolent CTCL characterized by its chronic, persistent, and relapsing course; thus, long-term followup focusing on the EFS is crucial for evaluating the effects of therapies in the treatment of focally recalcitrant MF. Our real-world data demonstrated the long-term followup outcomes in EFS and events in the 15 focally recalcitrant MF patients (Table 2) treated with intralesional interferon injection in daily clinical practice. The median EFS was 4.0 months (95\% CI, 1.8-6.1 months; range, 1-28+ months). Events included discontinuation of intralesional injection due to significant improvement $(13.3 \%, 2 / 15)$ or lack of response (20.0\%, 3/15), local recurrence $(6.7 \%, 1 / 15)$, and distant recurrence $(33.3 \%, 5 / 15)$. In our study $90.0 \%(9 / 10)$ of the CR patients experienced no local recurrence, while patient 7 (10.0\%, 1/10) had local recurrence, and patients 5, 8, 11, 14, and $15(50.0 \%, 5 / 10)$ had distant recurrence. By contrast, the previous literature reported no local recurrence $(18,19,26-29)$, and 1 case of distant recurrence (patient 2, 16.7\%, 1/6) was reported by Vonderheid et al. (18). These differences in the rate of local and distant recurrence might result from the longer follow-up time in our study, the drug-resistance of the disease in these patients, and the relapsing course of MF. Comparative studies and longer follow-up times are needed to investigate the recurrence rate, time, and relevant clinical and pathological factors.

In general, flu-like symptoms, such as fever, fatigue, arthralgia, and headache, are the most common acute AEs associated with IFN- $\alpha$. The most common chronic AEs include fatigue, anorexia, and weight loss. Other noteworthy side effects are cytopenia, liver toxicity, depressed mood, and increased irritability. In our study, influenza-like symptoms were the most frequently reported drug-related AEs, with 13 (86.7\%) patients experiencing fever and $10(66.7 \%)$ experiencing fatigue. Lab test abnormalities occurred in 3 patients, 2 had elevated liver function enzyme levels, and 1 had leukocytopenia. All of these AEs were mild to moderate and either resolved after the therapy ended or, if necessary, were controlled using acetaminophen. Compared with interferon intramuscular injection, intralesional injection led to milder AEs in $26.7 \%$ (4/15) of patients. Our findings based on longer follow-up time (mean, 18.9 months; range, 4-32 months) revealed that intralesional interferon injection was well tolerated, with mild-to-moderate AEs, mild and reversible changes in lab test results and no severe AEs, which was consistent with the AEs reported by Wolff et al. (19), Vonderheid et al. (18), Qiu et al. (26), Hu et al. (27), and Dummer et al. (29). These results evince the safety and tolerability of interferon intralesional injection in MF patients.

Currently, MF are generally considered to be incurable. For relapsed/refractory MF that is resistant to conventional therapies, treatment can be especially challenging. In this study, the rate of CR and the rate of objective response (OR) were $66.7 \%$ and $73.3 \%$, respectively; $77.8 \%$ (7/9) of patients who had $\geq 3$ failed therapies reached CR. In patients with MF that had shown resistance to phototherapy, intramuscular interferon and oral acitretin, $83.3 \%(5 / 6)$ achieved CR, and the other $16.7 \%(1 / 6)$ had PR. The AEs were tolerable and reversible. The efficacy and safety of treatment for relapsed or refractory MF and SS in previous studies were shown in Table $3(21,23,26,27$, 30-39). The rates of CR and PR in our study tended to be higher, which might result from the lower MF stages and the localized therapeutic effects of intralesional injection. However, the EFS in our study tended to be shorter, and this might reveal the advantage in systemic effects of the systemic treatment listed in Table 3. The milder AEs in our study indicated intralesional interferon injection could be well tolerated, and might have an advantage in safety and patient adherence. These encouraging results showed the effectiveness and safety of interferon intralesional injection in treatment for focally resistant MF.

The present study had some limitations. First, it was a single-center study focused on a small number of patients with focally recalcitrant MF treat with intralesional IFN- $\alpha$ 2a. The relatively small sample size might not sufficiently address the heterogeneities shown in the real-world data. However, because focally recalcitrant MF is rare, we consider that our long-term follow-up data and findings regarding the effectiveness and safety of the treatment in 


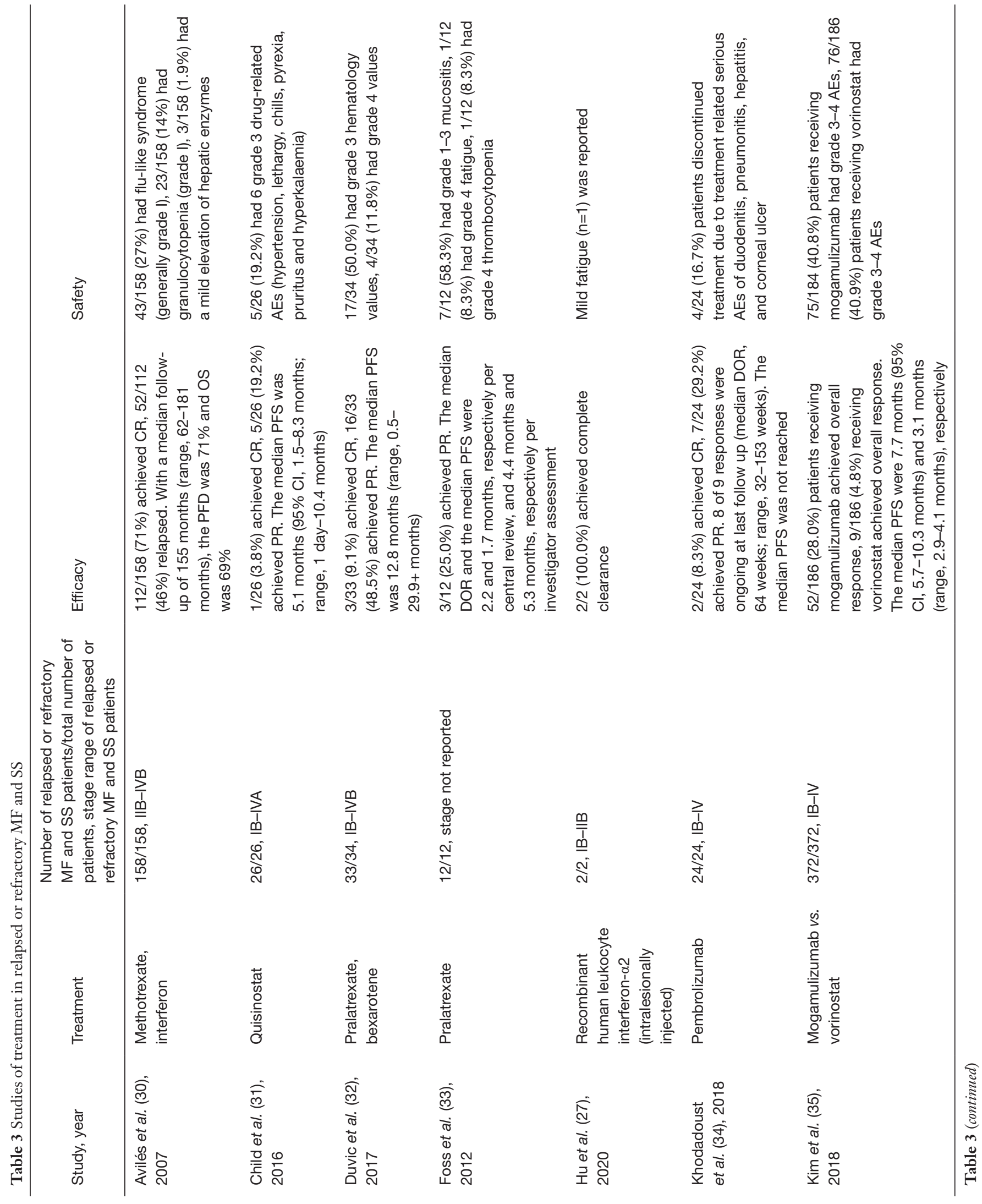




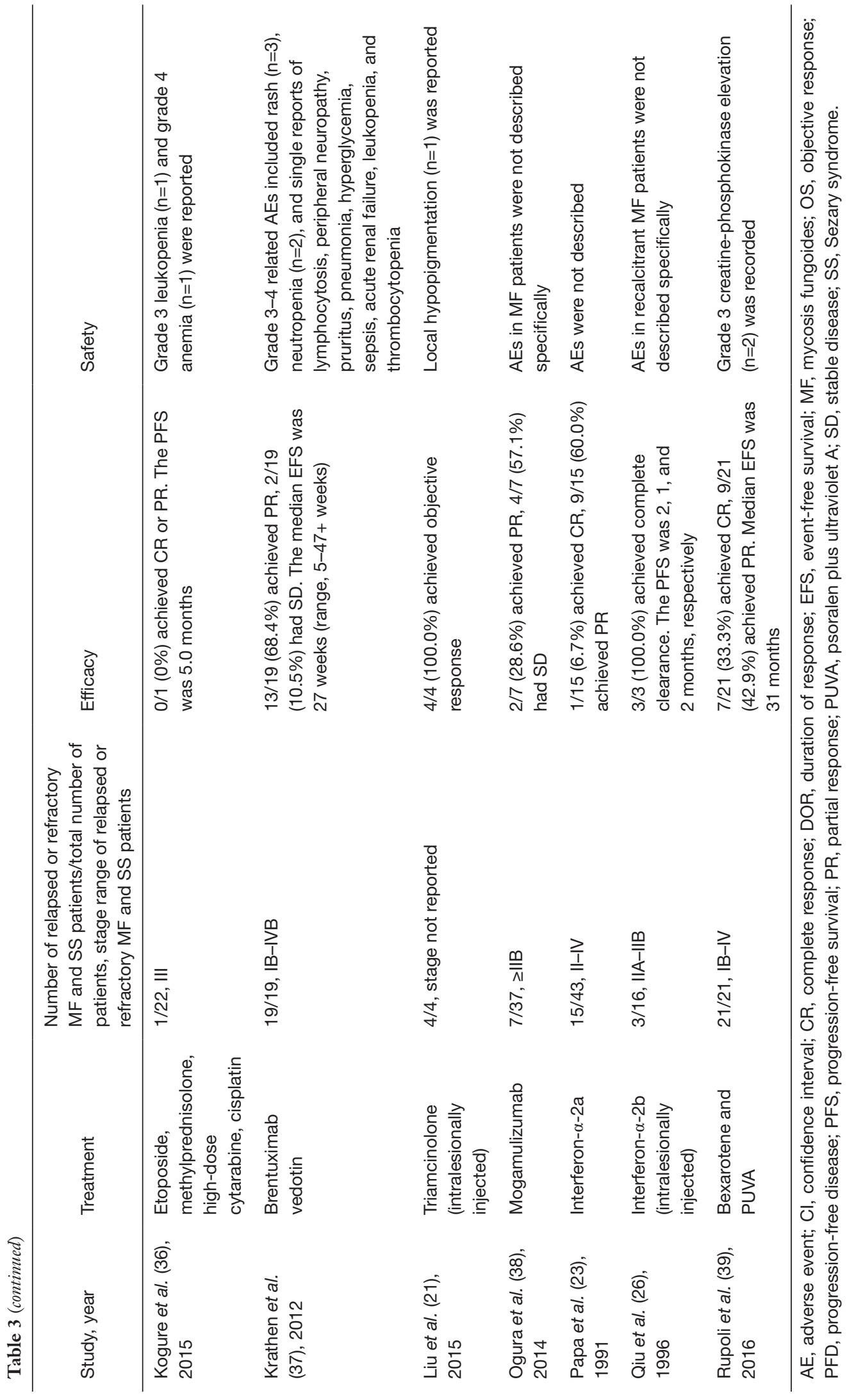


this specific patient population nevertheless have clinical importance when interpreted cautiously. Second, owing to the rarity of focally recalcitrant $\mathrm{MF}$, there was no control group and the therapeutic effects were compared with each patient's historical treatment. Third, due to the retrospective design of our study, missing and incomplete data, and charts of lower quality was inevitable, and this might have affected our results.

\section{Conclusions}

In conclusion, interferon intralesional injection is safe and effective for the treatment of focally resistant MF, especially in lesions in sun-exposed areas and in the patients with a disease duration lasting less than 20 years. This promising treatment modality may represent an effective, tolerable, and feasible therapeutic option for refractory MF lesions. On the basis of real-world data, our study has brought insights into interferon intralesional injection treatment in focally resistant MF.

\section{Acknowledgments}

We thank the AME Editing Service (http://editing. amegroups.com) for its linguistic assistance in editing the English text of a draft of this manuscript.

Funding: This work was supported by the grants from the National Scientific Data Sharing Platform for Population and Health - Clinical Center [NCMI-ABS03-201809-03 to YHL], and Peking Union Medical College Postgraduate Innovation Fund [2019-1002-72 to SYZ].

\section{Footnote}

Reporting Checklist: The authors have completed the STROBE reporting checklist. Available at http://dx.doi. org/10.21037/atm-20-1458

Data Sharing Statement: Available at http://dx.doi. org/10.21037/atm-20-1458

Conflicts of Interest: All authors have completed the ICMJE uniform disclosure form (available at http://dx.doi. org/10.21037/atm-20-1458). The authors have no conflicts of interest to declare.

Ethical Statement: The authors are accountable for all aspects of the work in ensuring that questions related to the accuracy or integrity of any part of the work are appropriately investigated and resolved. The study was conducted in accordance with the Declaration of Helsinki (as revised in 2013). The study was approved by the institutional ethics committee of Peking Union Medical College Hospital (No. S-k357) and informed consent was taken from all the patients.

Open Access Statement: This is an Open Access article distributed in accordance with the Creative Commons Attribution-NonCommercial-NoDerivs 4.0 International License (CC BY-NC-ND 4.0), which permits the noncommercial replication and distribution of the article with the strict proviso that no changes or edits are made and the original work is properly cited (including links to both the formal publication through the relevant DOI and the license). See: https://creativecommons.org/licenses/by-nc-nd/4.0/.

\section{References}

1. Wong HK, Mishra A, Hake T, et al. Evolving insights in the pathogenesis and therapy of cutaneous T-cell lymphoma (mycosis fungoides and Sezary syndrome). Br J Haematol 2011;155:150-66.

2. Wilcox RA. Cutaneous T-cell lymphoma: 2011 update on diagnosis, risk-stratification, and management. Am J Hematol 2011;86:928-48.

3. Wong HK. Novel biomarkers, dysregulated epigenetics, and therapy in cutaneous T-cell lymphoma. Discov Med 2013;16:71-8.

4. Herne KL, Talpur R, Breuer-McHam J, et al. Cytomegalovirus seropositivity is significantly associated with mycosis fungoides and Sezary syndrome. Blood 2003;101:2132-6.

5. Chang YT, Liu HN, Chen CL, et al. Detection of EpsteinBarr virus and HTLV-I in T-cell lymphomas of skin in Taiwan. Am J Dermatopathol 1998;20:250-4.

6. Guenova E, Watanabe R, Teague JE, et al. TH2 cytokines from malignant cells suppress TH1 responses and enforce a global TH2 bias in leukemic cutaneous T-cell lymphoma. Clin Cancer Res 2013;19:3755-63.

7. Wysocka M, Benoit BM, Newton S, et al. Enhancement of the host immune responses in cutaneous T-cell lymphoma by CpG oligodeoxynucleotides and IL-15. Blood 2004;104:4142-9.

8. Yoo EK, Cassin M, Lessin SR, et al. Complete molecular remission during biologic response modifier therapy for Sezary syndrome is associated with enhanced helper $\mathrm{T}$ 
type 1 cytokine production and natural killer cell activity. J Am Acad Dermatol 2001;45:208-16.

9. Spaccarelli N, Rook AH. The Use of Interferons in the Treatment of Cutaneous T-Cell Lymphoma. Dermatol Clin 2015;33:731-45.

10. Furudate S, Fujimura T, Kakizaki A, et al. Tumorassociated M2 macrophages in mycosis fungoides acquire immunomodulatory function by interferon alpha and interferon gamma. J Dermatol Sci 2016;83:182-9.

11. Tanita K, Fujimura T, Sato Y, et al. Bexarotene Reduces Production of CCL22 From Tumor-Associated Macrophages in Cutaneous T-Cell Lymphoma. Front Oncol 2019;9:907.

12. Fujimura T, Kambayashi Y, Fujisawa Y, et al. TumorAssociated Macrophages: Therapeutic Targets for Skin Cancer. Front Oncol 2018;8:3.

13. Jumbou O, N'Guyen JM, Tessier MH, et al. Long-term follow-up in 51 patients with mycosis fungoides and Sezary syndrome treated by interferon-alfa. Br J Dermatol 1999;140:427-31.

14. McGinnis KS, Junkins-Hopkins JM, Crawford G, et al. Low-dose oral bexarotene in combination with lowdose interferon alfa in the treatment of cutaneous T-cell lymphoma: clinical synergism and possible immunologic mechanisms. J Am Acad Dermatol 2004;50:375-9.

15. Rupoli S, Goteri G, Pulini S, et al. Long-term experience with low-dose interferon-alpha and PUVA in the management of early mycosis fungoides. Eur J Haematol 2005;75:136-45.

16. Wagner AE, Wada D, Bowen G, et al. Mycosis fungoides: the addition of concurrent and adjuvant interferon to total skin electron beam therapy. Br J Dermatol 2013;169:715-8.

17. Jimbow K, Yamana K, Ishida O, et al. Evaluation of rIFNgamma in the treatment of lymphoma and melanoma of the skin by systemic and intralesional administration. Gan To Kagaku Ryoho 1987;14:152-8.

18. Vonderheid EC, Thompson R, Smiles KA, et al. Recombinant interferon alfa-2b in plaque-phase mycosis fungoides. Intralesional and low-dose intramuscular therapy. Arch Dermatol 1987;123:757-63.

19. Wolff JM, Zitelli JA, Rabin BS, et al. Intralesional interferon in the treatment of early mycosis fungoides. J Am Acad Dermatol 1985;13:604-12.

20. Olsen EA, Whittaker S, Kim YH, et al. Clinical end points and response criteria in mycosis fungoides and Sezary syndrome: a consensus statement of the International Society for Cutaneous Lymphomas, the United States
Cutaneous Lymphoma Consortium, and the Cutaneous Lymphoma Task Force of the European Organisation for Research and Treatment of Cancer. J Clin Oncol 2011;29:2598-607.

21. Liu DY, Shaath T, Rajpara AN, et al. Safe and efficacious use of intralesional steroids for the treatment of focally resistant mycosis fungoides. J Drugs Dermatol 2015;14:466-71.

22. Ross C, Tingsgaard P, Jorgensen $\mathrm{H}$, et al. Interferon treatment of cutaneous T-cell lymphoma. Eur J Haematol 1993;51:63-72.

23. Papa G, Tura S, Mandelli F, et al. Is interferon-alpha in cutaneous T-cell lymphoma a treatment of choice. Br J Haematol 1991;79:48-51.

24. Hüsken AC, Tsianakas A, Hensen P, et al. Comparison of pegylated interferon alpha-2b plus psoralen PUVA versus standard interferon alpha-2a plus PUVA in patients with cutaneous T-cell lymphoma. J Eur Acad Dermatol Venereol 2012;26:71-8.

25. Doerschner M, Pekar-Lukacs A, Messerli-Odermatt O, et al. Interferon alfa-2a maintenance after salvage autologous stem cell transplantation in atypical mycosis fungoides with central nervous system involvement. Br J Dermatol 2019;181:1296-302.

26. Qiu B, Chen M. Treatment of cutaneous T cell lymphoma with low doses of interferon alpha-2b. Chin Med J (Engl) 1996;109:404-6.

27. Hu JK, Carlson K, Girardi M. Low-Dose Intralesional Recombinant Interferon-alpha2b in the Treatment of Mycosis Fungoides. Yale J Biol Med 2020;93:41-4.

28. Yamamoto T, Sasaki G, Sato T, et al. Cytokine profile of tumor cells in mycosis fungoides: successful treatment with intra-lesional interferon-gamma combined with chemotherapy. J Dermatol 1995;22:650-4.

29. Dummer R, Eichmuller S, Gellrich S, et al. Phase II clinical trial of intratumoral application of TG1042 (adenovirus-interferon-gamma) in patients with advanced cutaneous T-cell lymphomas and multilesional cutaneous B-cell lymphomas. Mol Ther 2010;18:1244-7.

30. Avilés A, Nambo MJ, Neri N, et al. Interferon and low dose methotrexate improve outcome in refractory mycosis fungoides/Sezary syndrome. Cancer Biother Radiopharm 2007;22:836-40.

31. Child F, Ortiz-Romero PL, Alvarez R, et al. Phase II multicentre trial of oral quisinostat, a histone deacetylase inhibitor, in patients with previously treated stage IBIVA mycosis fungoides/Seezary syndrome. Br J Dermatol 2016;175:80-8. 


\section{Page 14 of 14}

32. Duvic M, Kim YH, Zinzani PL, et al. Results from a Phase I/II Open-Label, Dose-Finding Study of Pralatrexate and Oral Bexarotene in Patients with Relapsed/ Refractory Cutaneous T-cell Lymphoma. Clin Cancer Res 2017;23:3552-6.

33. Foss F, Horwitz SM, Coiffier B, et al. Pralatrexate Is an Effective Treatment for Relapsed or Refractory Transformed Mycosis Fungoides: A Subgroup Efficacy Analysis From the PROPEL Study. Clin Lymphoma Myeloma Leuk 2012;12:238-43.

34. Khodadoust MS, Rook A, Porcu P, et al. Durable Responses with Pembrolizumab in Relapsed/Refractory Mycosis Fungoides and Sezary Syndrome: Final Results from a Phase 2 Multicenter Study. Blood 2018;132.

35. Kim YH, Bagot M, Pinter-Brown L, et al. Mogamulizumab versus vorinostat in previously treated cutaneous T-cell lymphoma (MAVORIC): an international, open-label, randomised, controlled phase 3 trial. Lancet Oncol

Cite this article as: Zhang SY, Liu ZR, Yang L, Wang T, Liu J, Liu YH, Fang K. Real-world data on the effectiveness and safety of interferon-alpha-2a intralesional injection for the treatment of focally recalcitrant mycosis fungoides. Ann Transl Med 2020;8(15):920. doi: 10.21037/atm-20-1458

\section{Zhang et al. Intralesional interferon for focally recalcitrant MF}

2018;19:1192-204.

36. Kogure Y, Yoshimi A, Ueda K, et al. Modified ESHAP regimen for relapsed/refractory $\mathrm{T}$ cell lymphoma: a retrospective analysis. Ann Hematol 2015;94:989-94.

37. Krathen M, Sundram U, Bashey S, et al. Brentuximab vedotin demonstrates significant clinical activity in relapsed or refractory mycosis fungoides with variable CD30 expression. Blood 2012;120.

38. Ogura M, Ishida T, Hatake K, et al. Multicenter phase II study of mogamulizumab (KW-0761), a defucosylated anti-cc chemokine receptor 4 antibody, in patients with relapsed peripheral T-cell lymphoma and cutaneous T-cell lymphoma. J Clin Oncol 2014;32:1157-63.

39. Rupoli S, Canafoglia L, Goteri G, et al. Results of a prospective phase II trial with oral low-dose bexarotene plus photochemotherapy (PUVA) in refractory and/or relapsed patients with mycosis fungoides. Eur J Dermatol 2016;26:13-20. 